\title{
Analysis of the Chinese Airline Network as a multi-layer network
}

\author{
Wen-Bo Du ${ }^{\mathrm{a}, \mathrm{b}, \mathrm{c}, *}$, Xing-Lian Zhou ${ }^{\mathrm{a}, \mathrm{b}, \mathrm{c}}$, Oriol Lordan ${ }^{\mathrm{d}}$, Zhen Wang ${ }^{\mathrm{e}}$, Chen \\ Zhao $^{\mathrm{f}}$, Yan-Bo Zhu ${ }^{\mathrm{a}, \mathrm{b}, \mathrm{f}}$ \\ ${ }^{a}$ School of Electronic and Information Engineering, Beihang University,Beijing 100191, \\ P.R. China \\ ${ }^{b}$ National Key Laboratory of CNS/ATM, Beijing 100191, P.R. China \\ ${ }^{c}$ Beijing Laboratory for General Aviation Technology, Beijing 100191, P.R. China \\ ${ }^{d}$ Universitat Politècnica de Catalunya-BarcelonaTech, C/Colom no. 11, Terrassa 08222, \\ Spain \\ ${ }^{e}$ Interdisciplinary Graduate School of Engineering Sciences, Kyushu University, \\ Kasuga-koen, Kasuga-shi, Fukuoka 816-8580, Japan \\ ${ }^{f}$ Aviation Data Communication Corporation, Beijing 100191, P.R. China
}

\begin{abstract}
This paper encapsulates the Chinese Airline Network (CAN) into multi-layer infrastructures via the "k-core decomposition" method. The network is divided into three layers: Core layer, containing airports of provincial capital cities, is densely connected and sustains most flight flow; Bridge layer, consisting of airports in Tier 2 and Tier 3 cities, mainly connects two other layers; and Periphery layer, comprising airports of remote areas, sustains little flight flow. Moreover, it is unveiled that CAN stays the most robust when low-degree nodes or high flight flow links are removed, which is similar to the Worldwide Airline Network (WAN), albeit less redundant.

Keywords: air transport network, Chinese Airline Network, k-core decomposition
\end{abstract}

\section{Introduction}

Given the important role of the transportation system for the modern society, transportation problems have attracted much attention, both theoretical

\footnotetext{
* Corresponding author

Email address: wenbodu@buaa.edu.cn (Wen-Bo Du)
}

Preprint submitted to Transportation Research Part E: Logistics and Transportation ReviewMay 17,2016 
and experimental [1]. The study of four typical means of transport: roadway, 5 railway, shipping and airway, has also permeated a myriad of scientific disciplines [2]. Though the contribution of airway is quite small compared with the other three, it has three significant advantages: (i) speed: many jet planes' cruise speed is faster than $850 \mathrm{~km} / \mathrm{h}$. The reduced travel time can keep perishable goods from decaying, can deliver goods such as newspapers or first-aid medicines on time, and can save passengers' valuable time. (ii) safety: the accident rate of air transport is still among the lowest. (iii) cost-saving: on the one hand, companies can reduce inventory level and accelerate turnover via fast air transport. On the other hand, air companies often provide less complex packaging and lower insurance expense. These elements can reduce companies' implicit cost. Due to the acceleration of the globalization process, the air transport system plays an increasingly more critical role in local, national, and international economies $[3$ and scientists from different communities pay special attention to the air transport infrastructure.

Complex network theory is naturally a useful tool to investigate the transport infrastructures. During the last decade, complex network theory has been widely applied to different transport methods, including urban traffic [4, 5], railway [6], subway [7, 8, and especially the air transport system [9, 10, 11, 12, 13, 14]. A great variety of publications have unveiled the topological structure and the dynamic behaviour of the air transport network, where airports are denoted by nodes and flights between airports are denoted by edges. For example, the World Airline Network (WAN) has been extensively studied. Barrat et al. 15] studied the correlations between weighted quantities of the WAN and found a strong correlation between the traffic flow and the network's topological properties. Colizza et al. developed a model to simulate the mobility of individuals from an so airport to another and found the important role of air transport network for the global pattern of emerging diseases [16]. Guimera et al. investigated the network of flight segments between city-pairs [17. A remarkable result they present is that the most connected nodes in the WAN are not necessarily the most central nodes, which means critical locations might not coincide with highly-connected 
hubs.

Since the national air route network is often associated with the economy and image of a country, it has been also extensively studied. For instance, Gautreau et al. studied the US airport network during the period from 1990 to 2000 and it is shown that an intense activity takes place at the local level though 40 the statistical distributions of most indicators are stationary [9]. Bagler et. al studied the Airport Network of India which is a small-world network with a truncated power-law distribution and signature of hierarchy feature [13. Rocha investigated the structure and evolution of the Brazilian airport route network and found that it has shrinked at the route level but it has growed in number passengers and amount of cargo [14]. Especially, as the largest developing country and the most active economy in the world, the aviation industry of China has undergone a rapid development in the past few decades. Now the Chinese air transport system ranks only second to the US and there are 25 airports with more than ten million annual passenger movements. Consequently, the Chinese Airline Network (CAN) has been extensively studied on its topology, traffic dynamic and evolution [10, 11, 12.

However, most of the previous research ignores the multi-layer nature of real systems. In fact, a multiplex model fits the real situation better, as it can define more accurately how the different dynamics develop in each layer 55 of a complex system. Therefore, the concepts strongly related to multiplex networks have been introduced into the study of air transport networks. Cardillo et. al [18] established the European Air Transport Network (EATN) where 15 biggest airline companies in Europe are considered as 15 layers. These authors found that the multi-layer structure strongly reduces the resilience of the system. Verma et. al [19] illustrated three distinct layers of the WAN based on the k-core decomposition and found that the WAN is a redundant and resilient network for long distance air travel, but otherwise breaks down completely with the removal of short and apparently insignificant connections.

Inspired by these works, we want to analyse if a regional network such as the Chinese Airline Network (CAN) can be described as a multi-layer network. 
For this purpose, we will analyse each layer and identify its particularities given the unique demographic and geographical properties of China. Furthermore, we will look at which airports and connections have the most important role in maintaining the CAN completely connected.

The paper is organized as follows. The following section makes detailed discussions on the topological properties of CAN. Afterwards, the robustness of CAN is analyzed and finally, conclusions are presented.

\section{Topological properties of the CAN}

The Chinese Airline Network (CAN) comprises all domestic flights within China scheduled in 2015 provided by the Civil Aviation Administration of China (CAAC). We define the CAN as an unweighted and undirected network where nodes are airports and there is an edge between two airports if they are connected directly, as in 17, 20, 21, 22.

Following this definition, the CAN has $N=203$ nodes (i.e. airports) and so $E=1877$ edges (i.e. connections) between airports. We define a binary adjacency matrix $A(N \times N)$ where $a_{i j}=1$ if there is a direct connection between airport $\mathrm{i}$ and $\mathrm{j}$, otherwise $a_{i j}=0$. The degree of a node is the number of connections of that node and it is defined as $k_{i}=\sum_{j=0}^{N} a_{i j}$. The CAN exhibits a two-regime power-law degree distribution with two different exponents as in refs. [10, 11, 12. The average degree of the CAN is about $\langle k\rangle=18.48$. The top 3 airports with the highest degree are Beijing $(k=136)$, Shanghai $(k=120)$ and Guangzhou $(k=100)$. The average cluster coefficient is 0.73 and the average path length is 2.19, indicating the CAN is a typical small-world network. The diameter of the CAN is 5 and over $98 \%$ of the routes can be covered with at most 3 connections. To investigate flight flow on the CAN, the weighted network is defined as $W(N \times N) . w_{i j}=f_{i j}$ if $a_{i j}=1$, otherwise $w_{i j}=0$ where $f_{i j}$ is the number of direct flights per week between airport $i$ and airport $j$. Thus the strength of a node is defined as $s_{i}=\sum_{j=0}^{j=N} w_{i j} a_{i j}$. The top 3 airports with the largest flight flow are also Beijing, Shanghai and Guangzhou. Figure 1 
Chinese civil aviation industry is quite unbalanced: most airlines and airports are located in eastern China.

The analysis of a single air transport network has enabled us to understand the rationale behind its structure. Nevertheless, the traditional methods fail to uncover the underlying multi-layer property of air transport networks. Therefore, we will analyse the CAN via the k-core decomposition algorithm [19, 23]. The steps of the k-core decomposition are:

- First, all nodes with $k_{i}=1$ are removed from the CAN. These nodes form the network Periphery. Some nodes may get completely disconnected from layers cannot provide clear results or conclusions to audience. To simplify the layer structure, $\mathrm{ki}=1$ is defined as the bottom layer, like ([19]). From Fig. 2 , one can see that the Core layer is located in eastern China, and that it is densely connected and bears most of the flight flow. The Bridge layer, in which the airports are evenly located, is sparser. For the Periphery layer, the airports are more remote and there is no direct flight between them at all. Next, we will analyse the properties of each layer and observe how geography and demographics have defined the CAN. 


\subsection{Core layer: Capital cities}

the CAN. Most airports are located in the provincial capital cities, except for Qingdao, Xiamen, Wenzhou, Guilin, Sanya, Shenzhen and Ningbo, all of which are economically developed cities or tourist destinations in China. It's not surprising that the air transport demand is high in these cities. From Figure 2, of all airports in the Core layer is more than 900 flights per week. Moreover, there are 48748 flights ( $63.07 \%$ of the total traffic flow) whose departure airport and arrival airport are in the Core layer, 24179 flights $(31.98 \%$ of the total traffic flow) connected between the Core layer and the Bridge layer, and 197 flights

135 (only $0.25 \%$ of the total traffic flow) connected between Core layer and Periphery layer. Furthermore, top 12 airports with highest degree are listed in Table 1 . We can find that the flight flow of airports generally decreases with the decrement of $k$, indicating a positive correlation between degree and strength. On the contrary, the cluster coefficient increases as $k$ decreases. This can be explained with airports in the Core layer, while the connections between the Bridge and the Periphery are sparser (this will be shown in following discussions), resulting in large-degree hubs in the Core having fewer triangles and the cluster coefficients being relatively lower.

145 Core layer, we define $R_{a}^{i n}\left(R_{f}^{i n}\right)$ as the ratio of connections (flight flow per week) within the Core layer and $R_{a}^{\text {out }}\left(R_{f}^{o u t}\right)$ as the ratio of connections (flight flow per week) between the Core and the Bridge for an airport. Since the Periphery sustains very little flight flow, we don't take it into consideration here. In Figure

$R_{f}^{o u t}$ increase with the node degree. Only for the top 11 highest degree airports, $R_{a}^{\text {out }}$ is larger than $R_{a}^{\text {in }}$ (Figure $3(\mathrm{a})$ ), indicating that these 11 airports play the role of connecting the Bridge with the Core while the rest tend to connect with other airports within the Core layer. Figure $3(\mathrm{~b})$ shows that $R_{f}^{i n}$ is much 

airports in the Periphery or the Bridge, leading to its low cluster coefficient. In Figure 4, we can see that the $R_{a}^{i n}\left(R_{f}^{i n}\right)$ is smaller than $R_{a}^{\text {out }}\left(R_{f}^{\text {out }}\right)$ for the same airport. There are 43 airports whose $R_{a}^{i n}=0$, indicating they only have 

airports connect with Beijing airport, about 24 airports connect with Shanghai airport, 18 airports connect with Guangzhou airport and 16 airports connect with Shenzhen airport. In addition, just a few remote airports such as Altay, Tacheng, Hetian and Ali with $R_{a}^{i n}=1$ only have connections with Lasa and

Urumchi. So the airports with $R_{a}^{i n}=1$ or $R_{a}^{i n}=0$ make little contribution to the flight flow between the Core and the Bridge. On the contrary, other airports with $0<R_{a}^{i n}<1$ carry the most flight flow in this layer.

Figure 5 is drawn to help understand the relationship between the ratio of connections and the ratio of flight flow. In Figure $5(\mathrm{a})$ we can see that $R_{f}^{i n}$ is larger than $R_{a}^{i n}$ for most airports in the Core layer. Take Beijing as an example, about $26 \%$ of the connections in the Core layer sustain about $70 \%$ of the flight flow. On the contrary, $R_{f}^{\text {out }}$ is smaller than $R_{a}^{\text {out }}$ for most airports in the Core layer (Figure 5(b)). That is to say, the average flight flow for a connection within the Core layer is larger than that of a connection between the Core layer and the Bridge. However, there is no obvious correlation between $R_{f}^{\text {in }}\left(R_{f}^{\text {out }}\right)$ and $R_{a}^{i n}\left(R_{a}^{\text {out }}\right)$ for the airports in the Bridge layer in Figure 5(c) and Figure $5(\mathrm{~d})$.

\subsection{Periphery layer: Remote areas}

Finally, we look at the structure and properties of the airports in the Periphery layer. In this layer there are 15 airports that involve $7.40 \%$ of airports in the CAN. It is important to remember that here all airports have just 1 connection to other airports, i.e. $k_{i}=1$. Most airports are located in small cities of remote areas. Moreover, most airports only have connection with its provincial capital cities except for Jiujiang connecting with Shanghai airport. Figure 2 shows that there are no flights within this layer at all. And the airports in this layer tend to connect with the airports in the Bridge layer more than those in the Core layer. 


\section{CAN robustness}

It is important to analyse the robustness of the CAN in order to identify

215

nected. This is especially important in cases where interruption in the service of airports or routes might occur, be it due to sever weather or other security alerts. Even though these events usually happen at a local level, they may affect the overall performance of the whole transport system. Therefore, we will study CAN.

Firstly, we analyse the extreme case of sequentially shutting down airports. We remove airports from the network following a high-degree or low-degree selection strategy. Generally, $S(q)$, which represents the largest connected com$q$ is the fraction of airports being shut down. Figure 6(a) shows that upon removal of the highest degree airports, the size of the largest connected component drops significantly. The CAN disintegrates into many small clusters when the 28 highest-degree airports are removed, where the Core layer is not completely silient as the WAN, in which more than $80 \%$ of the airports remain connected when its Core layer is completely disconnected [19. This difference in resilience is likely to be related to the difference in the proportion of airports in the Core layer; $17.70 \%$ of the airports in the CAN are in its Core layer, while only $2.26 \%$ holds the Chinese Airline Network together. Furthermore, its Periphery layer does not possess a star-like structure like the one in the WAN, so when the most connected airports in the CAN's Core layer stop being functional, there are no airports in the Periphery or the Bridge that can keep a lot of airports connected. By contrast, the size of the largest cluster decays linearly using a low-degree strategy. This can be explained by most low-degree airports being located in the Periphery or the Bridge, so the removal of these airports does not 
affect the rest of the network.

After considering airport shutdowns, we focus on a more common scenario which involves flights being cancelled. The removal of connections using both a highest flight flow and a lowest flight flow strategy is studied in this paper. Figure 6(b) shows that the CAN is less robust in the low flight flow removing strategy than the high flight flow removing strategy, which is similar to what is found about the WAN [19. These results can be explained by the high flight flow connections occurring between highly connected airports. For this reason, the removal of high flight flow connections causes fewer airports to become completely disconnected from the network than with low flight flow connections. In this regard, the CAN is quite resilient upon removing the high flight flow connections.

255 Despite inspiring findings, these above mentioned outcomes are at the basis of two traditional, maybe oversimplified removal strategies. In reality, the highconnected nodes or connections may be not consistent with the most important functional ones ([24, 25]). The removal of modes or links may need to combine with other centrality measures of networks or the actual situations, such as betweenness, closeness or possible emergency, and the evaluation methods of robustness of networks may also go beyond the present framework. Thus, the robustness of CAN will be a project of great interest and deserves more attention in future studies.

\section{Conclusion}

In this paper, the Chinese Airline Network has been studied from the perspective of a multi-layer network. Using the k-core decomposition method, the CAN is divided into three layers, namely the Core layer, the Bridge layer and the Periphery layer. It is found that the Core is a densely connected network which sustains most of China's flight flow, and the Bridge layer plays the role of connecting the Core and the Periphery. We could observe how each layer consists of similar cities and areas, meaning that the CAN is a multi-layer network 
whose layers are defined by the geography and demography of China.

We also study the resilience of the CAN via the removal of nodes and connections. It is found that the CAN is not as redundant and resilient as the WAN in the high-degree targeted attack strategy. It disintegrates into many small clusters when 28 high-degree airports are removed, where the Core layer is not completely destroyed. This study may give a better understanding of the Chinese Airline Network from the perspective of a multi-layer network. Along the same framework, we also hope that it can inspire future study of some realistic issues, such as flight conflict resolution and flight schedule optimization.

\section{Acknowledgement}

This work is supported by the National Natural Science Foundation of China (Grant Nos. 61201314, 61221061 and 61231013) and Beijing Higher Education Young Elite Teacher Project (No. YETP1072).

\section{References}

[1] R. Faturechi, E. Miller-Hooks, Measuring the performance of transportation infrastructure systems in disasters: A comprehensive review, Journal of Infrastructure Systems 21 (1) (2015) 04014025. doi:10.1061/(ASCE) IS.1943-555X.0000212.

[2] D. Helbing, Traffic and related self-driven many-particle systems, Rev. Mod. Phys. 73 (2001) 1067-1141. doi:10.1103/RevModPhys.73.1067. URL http://link .aps .org/doi/10.1103/RevModPhys.73.1067

[3] G. Camelia, S. Mihai, The economic and social benefits of air transport, Ovidius University Annals, Economic Sciences Series 10 (1) (2010) 60-66.

295 [4] P. Crucitti, V. Latora, S. Porta, Centrality measures in spatial networks of urban streets, Physical Review E 73 (3) (2006) 036125. 
[5] S. Porta, P. Crucitti, V. Latora, The network analysis of urban streets: a dual approach, Physica A: Statistical Mechanics and its Applications 369 (2) (2006) 853-866.

[6] P. Sen, S. Dasgupta, A. Chatterjee, P. Sreeram, G. Mukherjee, S. Manna, Small-world properties of the indian railway network, Physical Review E 67 (3) (2003) 036106.

[7] V. Latora, M. Marchiori, Is the boston subway a small-world network?, Physica A: Statistical Mechanics and its Applications 314 (1) (2002) 109113.

[8] P. Angeloudis, D. Fisk, Large subway systems as complex networks, Physica A: Statistical Mechanics and its Applications 367 (2006) 553-558.

[9] A. Gautreau, A. Barrat, M. Barthélemy, Microdynamics in stationary complex networks, Proceedings of the National Academy of Sciences 106 (22) (2009) 8847-8852.

[10] W. Li, X. Cai, Statistical analysis of airport network of china, Physical Review E 69 (4) (2004) 046106.

[11] J. Zhang, X.-B. Cao, W.-B. Du, K.-Q. Cai, Evolution of chinese airport network, Physica A: Statistical Mechanics and its Applications 389 (18) (2010) 3922-3931.

[12] H. Liu, X. Zhang, L. Cao, B. Wang, T. Zhou, Analysis on the connecting mechanism of chinese city airline network, Sci. China Ser. G 39 (2009) 935.

[13] G. Bagler, Analysis of the airport network of india as a complex weighted network, Physica A: Statistical Mechanics and its Applications 387 (12) (2008) 2972-2980.

[14] L. E. da Rocha, Structural evolution of the brazilian airport network, Journal of Statistical Mechanics: Theory and Experiment 2009 (04) (2009) P04020. 
[15] A. Barrat, M. Barthelemy, R. Pastor-Satorras, A. Vespignani, The architecture of complex weighted networks, Proceedings of the National Academy of Sciences of the United States of America 101 (11) (2004) 3747-3752.

[16] V. Colizza, A. Barrat, M. Barthélemy, A. Vespignani, The role of the airline transportation network in the prediction and predictability of global epidemics, Proceedings of the National Academy of Sciences of the United States of America 103 (7) (2006) 2015-2020.

[17] R. Guimera, S. Mossa, A. Turtschi, L. N. Amaral, The worldwide air transportation network: Anomalous centrality, community structure, and cities' global roles, Proceedings of the National Academy of Sciences 102 (22) (2005) 7794-7799.

[18] A. Cardillo, M. Zanin, J. Gómez-Gardeñes, M. Romance, A. J. G. del Amo, S. Boccaletti, Modeling the multi-layer nature of the european air transport network: Resilience and passengers re-scheduling under random failures, The European Physical Journal Special Topics 215 (1) (2013) 23-33.

[19] T. Verma, N. A. Araújo, H. J. Herrmann, Revealing the structure of the world airline network, Scientific reports 4 .

[20] O. Lordan, J. M. Sallan, P. Simo, Study of the topology and robustness of airline route networks from the complex network approach: a survey and research agenda, Journal of Transport Geography 37 (2014) 112-120.

[21] O. Lordan, J. M. Sallan, P. Simo, D. Gonzalez-Prieto, Robustness of the air transport network, Transportation Research Part E: Logistics and Transportation Review 68 (2014) 155-163.

[22] O. Lordan, J. M. Sallan, P. Simo, D. Gonzalez-Prieto, Robustness of airline alliance route networks, Communications in Nonlinear Science and Numerical Simulation 22 (1) (2015) 587-595.

[23] S. N. Dorogovtsev, A. V. Goltsev, J. F. F. Mendes, K-core organization of complex networks, Physical review letters 96 (4) (2006) 040601. 
[24] S.-J. Wang, Z. Wang, T. Jin, S. Boccaletti, Emergence of disassortative mixing from pruning nodes in growing scale-free networks, Scientific Reports 4 (2014) 7536.

[25] S. Boccaletti, G. Bianconi, R. Criado, C. del Genio, J. Gmez-Gardees,

a M. Romance, I. Sendia-Nadal, Z. Wang, M. Zanin, The structure 360 and dynamics of multilayer networks, Physics Reports 544 (1) (2014) 1 - 122, the structure and dynamics of multilayer networks. doi:http://dx.doi.org/10.1016/j.physrep.2014.07.001. http://www.sciencedirect.com/science/article/pii/ S0370157314002105 


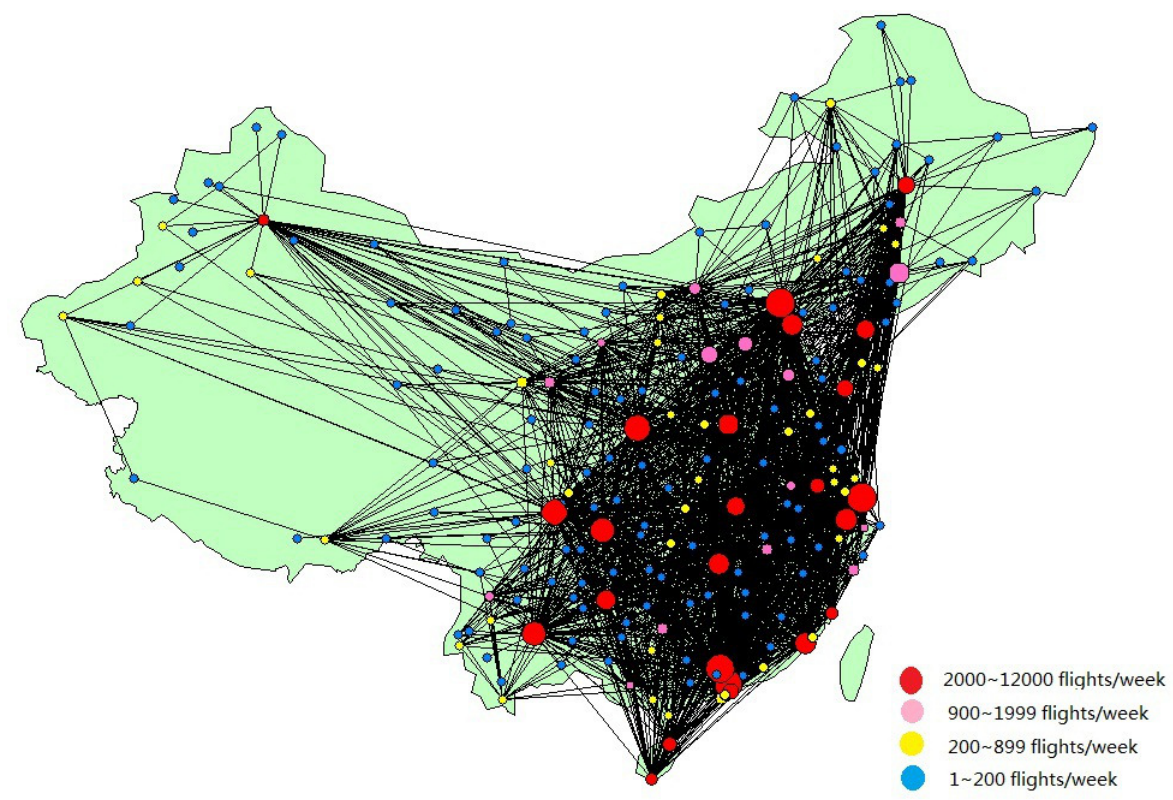

Figure 1: The Chinese Airline Network (CAN) contains 203 airports and 1877 connections. Here the size of the node is based on the degree of the airport and the nodes are coloured according to their flight flow. 


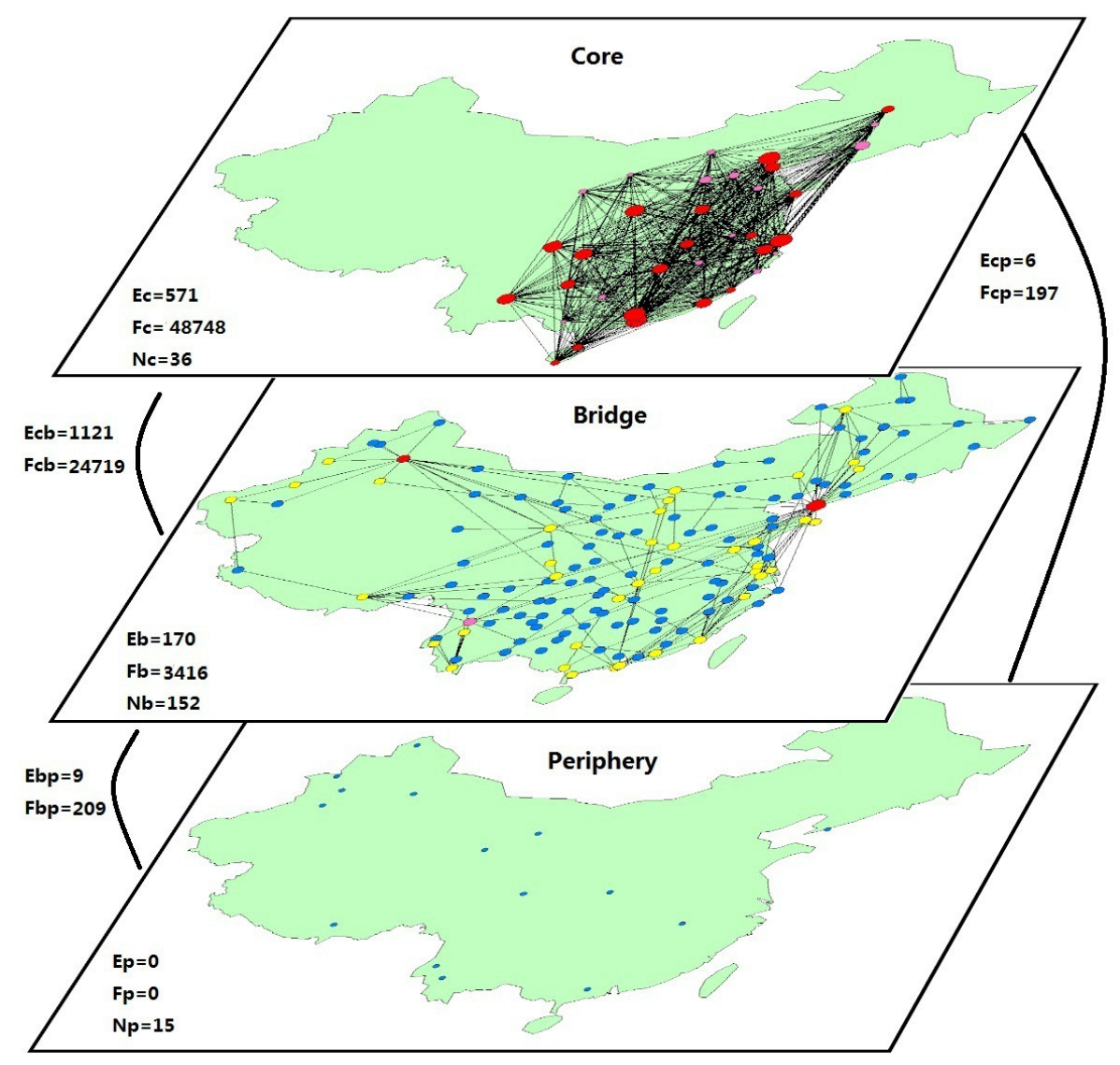

Figure 2: The Chinese Airline Network divided into three layers. The bottom layer is the Periphery with airports of $k_{i}=1$. The top layer is the Core with airports that form the nucleus of the k-core, $k_{i}^{\prime}=27$. The intermediate layer is the Bridge with all the remaining airports, which connect remote locations to hubs in the Core. It also shows the flight flow through the three layers. Here $F$ means the flight flow within or between layers, $E$ means the number of connections within or between layers, and $N$ means the number of nodes in each layer. 
Table 1: Top 12 airports with the largest degree in the Core layer. Here $K$ is the degree of an airport. $S$ is the strength (flight flow per week) of an airport. $C$ is the cluster coefficient of an airport. For a specific airport in the Core layer, $R_{a}^{i n}\left(R_{f}^{i n}\right)$ is the ratio of connections (flight flow per week) within the Core layer; $R_{a}^{o u t}\left(R_{f}^{o u t}\right)$ is the ratio of connections (flight flow per week) between the Core and Bridge.

\begin{tabular}{|c|c|c|c|c|c|c|c|}
\hline & $K$ & $S$ & $C$ & $R_{a}^{\text {in }}$ & $R_{a}^{\text {out }}$ & $R_{f}^{\text {in }}$ & $R_{f}^{\text {out }}$ \\
\hline Beijing/ZBAA & 136 & 11298 & 0.17 & 0.26 & 0.74 & 0.70 & 0.30 \\
\hline Shanghai/ZSSS & 120 & 11642 & 0.21 & 0.31 & 0.69 & 0.80 & 0.20 \\
\hline Guangzhou/ZGGG & 100 & 7036 & 0.26 & 0.34 & 0.66 & 0.80 & 0.20 \\
\hline Shenzhen/ZGSZ & 97 & 6408 & 0.28 & 0.37 & 0.63 & 0.83 & 0.17 \\
\hline Xi'an/ZLXY & 96 & 5551 & 0.26 & 0.37 & 0.63 & 0.71 & 0.29 \\
\hline Chengdu/ZUUU & 95 & 5784 & 0.26 & 0.38 & 0.62 & 0.72 & 0.28 \\
\hline Chongqing/ZUCK & 92 & 5212 & 0.30 & 0.40 & 0.60 & 0.77 & 0.23 \\
\hline Kunming/ZPPP & 85 & 5945 & 0.29 & 0.40 & 0.60 & 0.66 & 0.34 \\
\hline Hangzhou/ZSHC & 78 & 5094 & 0.32 & 0.42 & 0.58 & 0.83 & 0.17 \\
\hline Xiamen/ZSAM & 69 & 3614 & 0.41 & 0.48 & 0.52 & 0.83 & 0.17 \\
\hline Changsha/ZGHA & 69 & 3288 & 0.42 & 0.48 & 0.52 & 0.79 & 0.21 \\
\hline Tianjin/ZBTJ & 69 & 2673 & 0.40 & 0.48 & 0.52 & 0.78 & 0.22 \\
\hline
\end{tabular}



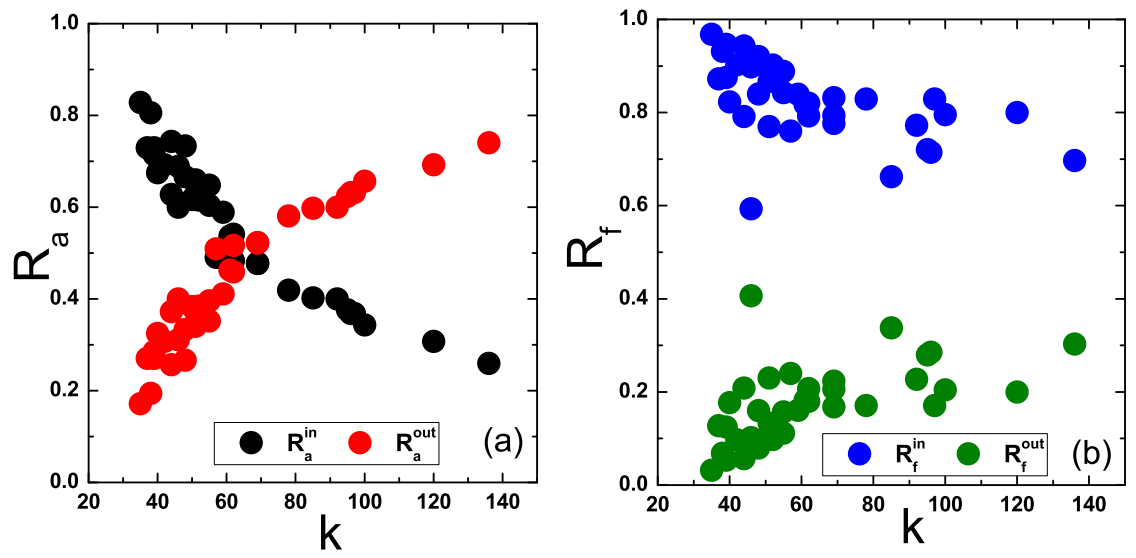

Figure 3: (colour online) (a) $R_{a}^{i n}$ (the ratio of connections within the Core layer, black dot) and $R_{a}^{\text {out }}$ (the ratio of connections between the Core and the Bridge, red dot) of the airports in the Core layer vs. the node degree. (b) $R_{f}^{i n}$ (the ratio of flight flow within the Core layer, blue dot) and $R_{f}^{\text {out }}$ (the ratio of flight flow between the Core and the Bridge, green dot) of the airports in the Core layer vs. node degree. 
Table 2: Top 11 airports with the highest degree in the Bridge layer. Here $\mathrm{K}$ means the degree of an airport. S means the strength of an airport. C means the cluster coefficient of an airport. $R_{a}^{i n}\left(R_{f}^{i n}\right)$ is the ratio of connections (flight flow) within the Bridge layer for an airport. $R_{a}^{\text {out }}\left(R_{f}^{\text {out }}\right)$ is the ratio of connections (flight flow) between the Core and Bridge for an airport.

\begin{tabular}{|c|c|c|c|c|c|c|c|}
\hline & $K$ & $S$ & $C$ & $R_{a}^{\text {in }}$ & $R_{a}^{\text {out }}$ & $R_{f}^{\text {in }}$ & $R_{f}^{\text {out }}$ \\
\hline Dalian/ZYTL & 59 & 2597 & 0.34 & 0.56 & 0.44 & 0.20 & 0.80 \\
\hline Urumchi/ZWWW & 44 & 3366 & 0.36 & 0.41 & 0.59 & 0.42 & 0.58 \\
\hline Zhuhai/ZGSD & 33 & 884 & 0.73 & 0.21 & 0.79 & 0.08 & 0.92 \\
\hline Xining/ZLXN & 32 & 817 & 0.63 & 0.30 & 0.70 & 0.20 & 0.80 \\
\hline Lijiang/ZPLJ & 30 & 1017 & 0.65 & 0.25 & 0.75 & 0.19 & 0.81 \\
\hline Shantou/ZGOW & 28 & 592 & 0.72 & 0.27 & 0.73 & 0.15 & 0.85 \\
\hline Quanzhou/ZSQZ & 28 & 776 & 0.64 & 0.30 & 0.70 & 0.14 & 0.86 \\
\hline Wuxi/ZSWX & 26 & 797 & 0.74 & 0.30 & 0.70 & 0.09 & 0.91 \\
\hline Mianyang/ZUMY & 25 & 384 & 0.84 & 0.20 & 0.80 & 0.18 & 0.82 \\
\hline Baotou/ZBOW & 24 & 434 & 0.83 & 0.18 & 0.82 & 0.16 & 0.84 \\
\hline Yantai/ZSYT & 24 & 989 & 0.75 & 0.18 & 0.82 & 0.11 & 0.89 \\
\hline
\end{tabular}



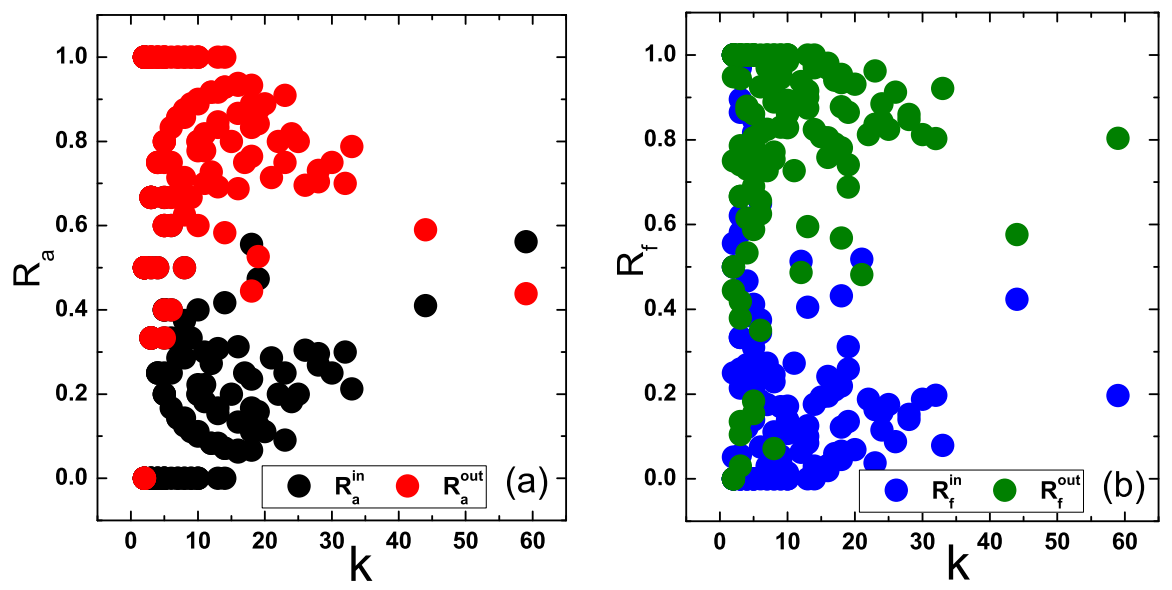

Figure 4: (colour online) (a) $R_{a}^{i n}$ (the ratio of connections within the Bridge layer, black dot) and $R_{a}^{\text {out }}$ (the ratio of connections between the Core and the Bridge, red dot) of the airports in the Bridge layer vs. node degree. (b) $R_{f}^{i n}$ (the ratio of flight flow within the Bridge layer, blue dot) and $R_{f}^{\text {out }}$ (the ratio of flight flow between Core and the Bridge, green dot) of the airports in the Bridge layer vs. node degree.
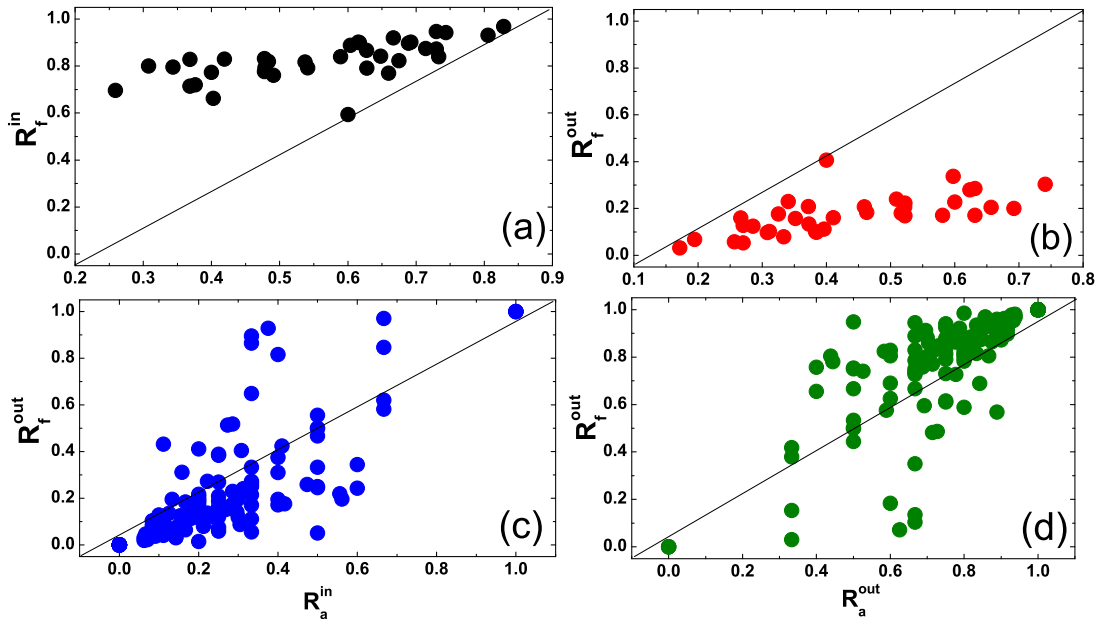

Figure 5: (colour online) (a) The relationship between $R_{a}^{i n}$ and $R_{f}^{i n}$ in the Core layer. (b) The relationship between $R_{a}^{\text {out }}$ and $R_{f}^{\text {out }}$ in the Core layer. (c) The relationship between $R_{a}^{\text {in }}$ and $R_{f}^{i n}$ in the Bridge layer. (d) The relationship between $R_{a}^{\text {out }}$ and $R_{f}^{\text {out }}$ in the Bridge layer. 

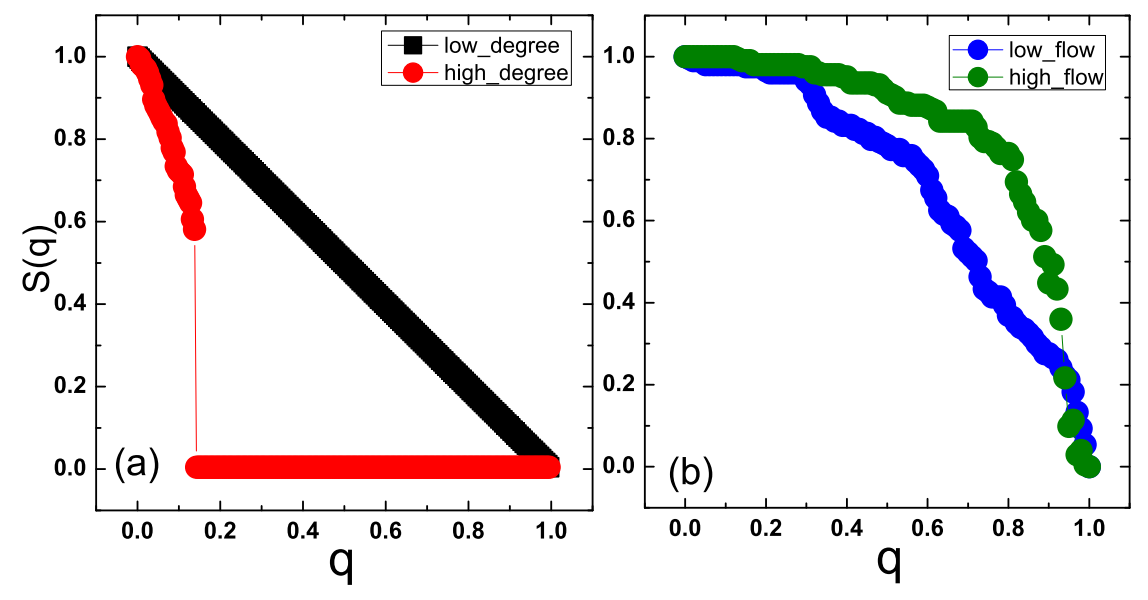

Figure 6: (colour online) (a) Drop in the size of the largest connected component of the CAN vs. nodes removed. High-degree (low-degree) refers to a conventional targeted removal strategy where a fraction q of nodes with the highest (lowest) degree is removed at each step. (b) Drop in the size of the largest connected component of the CAN vs connections removed. High-flow (low-flow) removal refers to the removal of all connections with the highest (lowest) flights up to a fraction q of nodes. 